\title{
The Accuracy of Self-report in Rheumatic Diseases
}

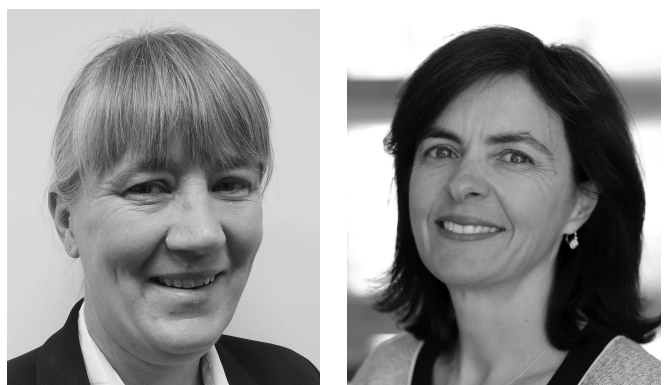

Determining knowledge of accurate population prevalence of rheumatic diseases is important in assessing the burden of the illness in the community and provides a basis for healthcare provision, policy, and workforce planning. The use of self-report is an integral part of determining the population prevalence of many chronic and non-registry-based diseases. Indeed, this is often the only way to obtain prevalence information for these conditions because definitive diagnostic tests may not exist or may be impractical to administer across a large number of people. Both prevalent and incident disease can be determined in this manner; however, there is evidence of a difference in the sensitivity of self-reporting prevalent and incident disease, and differences according to the disease examined. Oksanen, et $a l^{1}$ determined that the identification of true negatives was equally high for both prevalent and incident disease when compared with national registry data, but the sensitivity of incident ranged from 55\% to $63 \%$ compared with prevalent disease (78\%-96\%) for hypertension, diabetes, asthma, coronary heart disease, and rheumatoid arthritis (RA). Both prevalent and incident self-reported diabetes have also been shown over time by Schneider, et $\mathrm{al}^{2}$ to have $84 \%-97 \%$ specificity and $55 \%-80 \%$ sensitivity when compared with reference definitions (glucose and medication criteria).

The prevalence and incidence of inflammatory rheumatic conditions, in particular, is also often only measured using self-reported information, and because of the heterogeneity of diseases within this group, the information may or may not be supplemented and validated by medication data or other relevant clinical tests. A combination of self-report and other forms of validation has been used in various studies and has been demonstrated as a reliable means of determining disease prevalence, although the results can vary between different conditions. Broadly, self-reported arthritis has been demonstrated to have variable reliability, sensitivity, and specificity. In an older population, Bombard, et al compared self-reported arthritis using telephone and written surveys to rheumatological assessment. There was high reliability and moderate sensitivity and specificity of self-reported, doctor-diagnosed arthritis ${ }^{3}$. However, Lo, et al suggested caution with self-reported arthritis to determine prevalence because these authors demonstrated only moderate agreement between the self-report and the reference standard of musculoskeletal (MSK) signs or symptoms, and a sensitivity of $66.7 \%$ and specificity of $75.5 \%{ }^{4}$. Other studies have demonstrated good to excellent recall of self-reported, doctor-diagnosed arthritis using population surveys ${ }^{5,6}$.

More specifically, RA is one condition often examined in terms of the validity of self-report ${ }^{7,8,9}$, using self-report and/or validation. In African American women, self-reported RA along with the use of disease-modifying antirheumatic drugs demonstrated a high positive predictive value for RA ${ }^{7}$. However, Kvien, et $a l^{8}$ in 1996 suggested that patient self-report (whether diagnosed by a doctor and/or patient opinion) was unreliable when compared to clinical examination and advised caution in its use. More recently, Peeters, et $a l^{9}$ conducted a systematic review to determine the accuracy of self-reported RA [in addition to osteoarthritis (OA) and arthritis in general] in the population. Pooled results for RA demonstrated high specificity and sensitivity (as did those for $\mathrm{OA}$ ), with the gold standard being the American College of Rheumatology/European League Against Rheumatism recommendations, although other less optimal standards were also included in the review ${ }^{9}$. RA and systemic lupus erythematosus (SLE) have been examined by Walitt, et $a l^{10}$. While the accuracy levels of self-report alone for both RA and SLE were low (14.7\% and $11.8 \%$, respectively), they were improved by the addition of medication data for both conditions, with the positive predictive value increasing to $62.2 \%$ for RA and $40 \%$ for SLE. The authors concluded that self-report coupled with medication information was an effective means of confirming the presence of disease.

Gout is another form of inflammatory arthritis where

See Self-reported inflammatory arthritis, page 1134

Personal non-commercial use only. The Journal of Rheumatology Copyright @ 2017 . All rights reserved. 
doctor-diagnosed self-report has been found to be reliable. The gold standard definition of gout is the presence of monosodium urate crystals in synovial fluid analysis, or tophi ${ }^{11}$. However, this cannot be practically applied to large population-based studies, and in 2011, McAdams, et al ${ }^{12}$ examined the sensitivity and reliability of self-reported, physician-diagnosed gout in 2 population-based cohort studies. The self-report was examined against a standard of hospital discharge diagnosis of gout or the use of gout medication. The sensitivity, or proportion of true positives, was $84 \%$ and the reliability of self-report between questionnaires over time was high. The authors concluded that self-reported, doctor-diagnosed gout was appropriate to use to determine prevalence in epidemiological-based studies ${ }^{12}$.

All these issues around self-reporting the prevalence of inflammatory arthritis are highlighted in this issue of The Journal by Videm, et al, who report that the self-reported diagnosis of RA and ankylosing spondylitis (AS) in a Norwegian population often revealed false positives when validated against hospital case records, with verification in only $19.1 \%$ and $15.8 \%$, respectively ${ }^{13}$. The authors consider that the self-reported conditions are often false-positive and that validation requires using specialist case files or registry data. This represents one of the few studies to validate self-reported AS diagnosis. It is likely that the addition of self-reported medication data would have increased the sensitivity of this data.

However, there are other issues that need to be taken into consideration when obtaining self-reported information. Inaccuracy of self-report may be influenced by factors such as lack of communication by a medical professional to a patient regarding their diagnosis. Our previous work from a population-based survey demonstrated that over $50 \%$ of respondents with arthritis or gout had at risk/inadequate Functional Health Literacy (FHL), increasing to $70 \%$ of those self-reporting osteoporosis. After adjustment for age and sex, respondents in the arthritis subgroup of "don't know type of arthritis" and self-reported osteoporosis were significantly more likely to have inadequate FHL than the general population. This burden of low health literacy among these patients with arthritis, gout, and osteoporosis will affect responses to self-reported questions and influence the population estimates of these disorders ${ }^{14}$.

Another significant issue is question wording. Lack of consistency in case definition and variations across studies examining the prevalence of MSK disorders has been a longstanding issue for researchers and policymakers alike in this area ${ }^{15}$, as have issues related to response options. The provision of a response option that indicates, for example, that a study participant has arthritis, but does not know what type it is, may be valid in light of issues such as FHL, as described above. However, we have previously demonstrated that about $6 \%-10 \%$ of respondents may not know the type of arthritis that they have ${ }^{14,16}$. While this is a valid response, the inclusion of this option is not useful in determining the population prevalence of specific MSK conditions. This is supported by Krosnick, et al ${ }^{17}$, who suggest that these types of "no opinion" or "don't know" responses do not enhance data quality and may hamper meaningful measurements.

Self-report alone is generally a valid and reliable means of determining the prevalence of MSK disorders within the population when used appropriately within the acknowledged limitations. While it appears that some MSK diseases are more amenable to self-report than others, on a population level and within epidemiological studies, this may be the only means of obtaining any kind of prevalence data. Self-report can be improved by validating the information against other data collections (registries, medical records, or medications); however, this is not always practical or even appropriate for some MSK disorders (e.g., OA does not have specific medication regimens). The questions used, case definition, study population, and even FHL may all be involved in the responses obtained. But on the whole, self-report remains an integral component of determining the prevalence and incidence of MSK disorders in the community.

TIFFANY K. GILL, BAppSc, MAppSc, CertHlthEc, PGradDip(HlthSc), PGradDip(Biostats), MBA, PhD, Adelaide Medical School, The University of Adelaide;

CATHERINE L. HILL, MBBS, MD, MSc, FRACP, Staff Specialist, Rheumatology Unit, Rheumatology Department, The Queen Elizabeth Hospital, Woodville, South Australia, Australia, and Clinical Professor,

Adelaide Medical School, The University of Adelaide, Adelaide, South Australia, Australia.

Address correspondence to Dr. C.L. Hill, The Queen Elizabeth Hospital, Rheumatology, 28 Woodville Road, Woodville, South Australia 5011, Australia. E-mail: Catherine.Hill@sa.gov.au

\section{REFERENCES}

1. Oksanen T, Kivimäki M, Pentti J, Virtanen M, Klaukka T, Vahtera J Self-report as an indicator of incident disease. Ann Epidemiol 2010;20:547-54.

2. Schneider AL, Pankow JS, Heiss G, Selvin E. Validity and reliability of self-reported diabetes in the Atherosclerosis Risk in Communities study. Am J Epidemiol 2012;176:738-43.

3. Bombard JM, Powell KE, Martin LM, Helmick CG, Wilson WH. Validity and reliability of self-reported arthritis. Am J Prev Med 2005;28:251-8.

4. Lo T, Parkinson L, Cunich M, Byles J. Discordance between self-reported arthritis and musculoskeletal signs and symptoms in older women. BMC Musculoskelet Disord 2016;17:494.

5. Dal Grande E, Fullerton S, Taylor AW. Reliability of self-reported health risk factors and chronic conditions questions collected using the telephone in South Australia, Australia. BMC Med Res Methodol 2012;12:108.

6. Gill TK, Tucker GR, Avery JC, Shanahan EM, Menz HB, Taylor $\mathrm{AW}$, et al. The use of self-report questions to examine the prevalence of musculoskeletal problems: a test-retest study. BMC

Personal non-commercial use only. The Journal of Rheumatology Copyright @ 2017. All rights reserved. 
Musculoskelet Disord 2016;17:100.

7. Formica MK, McAlindon TE, Lash TL, Demissie S, Rosenberg L. Validity of self-reported rheumatoid arthritis in a large cohort: results from the Black Women's Health Study. Arthritis Care Res 2010;62:235-41.

8. Kvien TK, Glennås A, Knudsrød OG, Smedstad LM. The validity of self-reported diagnosis of rheumatoid arthritis: results from a population survey followed by clinical examinations. J Rheumatol 1996;23:1866-71.

9. Peeters GM, Alshurafa M, Schaap L, de Vet HC. Diagnostic accuracy of self-reported arthritis in the general adult population is acceptable. J Clin Epidemiol 2015;68:452-9.

10. Walitt BT, Constantinescu F, Katz JD, Weinstein A, Wang H, Hernandez RK, et al. Validation of self-report of rheumatoid arthritis and systemic lupus erythematosus: The Women's Health Initiative. J Rheumatol 2008;35:811-8.

11. Neogi T, Jansen TL, Dalbeth N, Fransen J, Schumacher HR, Berendsen D, et al. 2015 Gout classification criteria: an American College of Rheumatology/European League Against Rheumatism collaborative initiative. Ann Rheum Dis 2015;74:1789-98.

12. McAdams MA, Maynard JW, Baer AN, Köttgen A, Clipp S, Coresh $\mathrm{J}$, et al. Reliability and sensitivity of the self-report of physician-diagnosed gout in the campaign against cancer and heart disease and the atherosclerosis risk in the community cohorts.

J Rheumatol 2011;38:135-41.

13. Videm V, Thomas R, Brown MA, Hoff M. Self-reported diagnosis of rheumatoid arthritis or ankylosing spondylitis has low accuracy: data from the Nord-Trøndelag Health Study. J Rheumatol 2017;44:1134-41.

14. Hill CL, Appleton SL, Black J, Hoon E, Rudd RE, Adams RJ, et al. Role of health literacy in self-reported musculoskeletal disorders. Arthritis 2015;2015:607472.

15. Picavet HS, Hazes JM. Prevalence of self reported musculoskeletal diseases is high. Ann Rheum Dis 2003;62:644-50.

16. Gill TK, Hill CL, Adams RJ, Broderick D, Black J, Taylor AW. "I know I have arthritis but I don't know what type". Understanding and knowledge of this chronic condition. BMC Musculoskelet Disord 2010;11:174.

17. Krosnick JA, Holbrook AL, Berent MK, Carson RT, Hanemann WM, Kopp RJ, et al. The impact of "no opinion" response options on data quality: non-attitude reduction or an invitation to satisfice? Public Opin Q 2002;66:371-403.

J Rheumatol 2017;44:1109-11; doi:10.3899/jrheum.170564 